Internet Engineering Task Force (IETF)

G. Huston

Request for Comments: 6492

R. Loomans

Category: Standards Track

B. Ellacott

ISSN : 2070-1721

APNIC

R. Austein

ISC

February 2012

\title{
A Protocol for Provisioning Resource Certificates
}

Abstract

This document defines a framework for certificate management interactions between an Internet Number Resource issuer ("issuer") and an Internet Number Resource recipient ("subject") through the specification of a protocol for interaction between the two parties. The protocol supports the transmission of requests from the subject, and corresponding responses from the issuer encompassing the actions of certificate issuance, certificate revocation, and certificate status information reports. This protocol is intended to be limited to the application of Internet Number Resource Certificate management and is not intended to be used as part of a more general certificate management framework.

Status of This Memo

This is an Internet Standards Track document.

This document is a product of the Internet Engineering Task Force $(\mathrm{IETF})$. It represents the consensus of the IETF community. It has received public review and has been approved for publication by the Internet Engineering Steering Group (IESG). Further information on Internet Standards is available in Section 2 of RFC 5741.

Information about the current status of this document, any errata, and how to provide feedback on it may be obtained at http: //www.rfc-editor.org/info/rfc6492. 
Copyright Notice

Copyright (c) 2012 IETF Trust and the persons identified as the document authors. All rights reserved.

This document is subject to BCP 78 and the IETF Trust's Legal Provisions Relating to IETF Documents

(http://trustee.ietf.org/license-info) in effect on the date of publication of this document. Please review these documents carefully, as they describe your rights and restrictions with respect to this document. Code Components extracted from this document must include Simplified BSD License text as described in Section 4.e of the Trust Legal Provisions and are provided without warranty as described in the Simplified BSD License.

Table of Contents

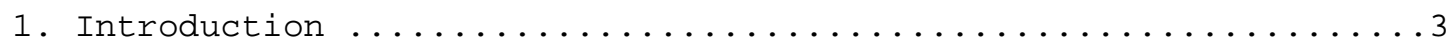

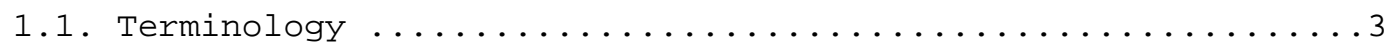

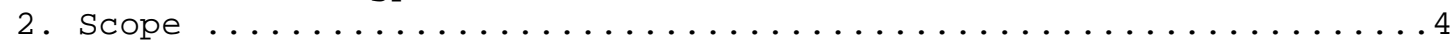

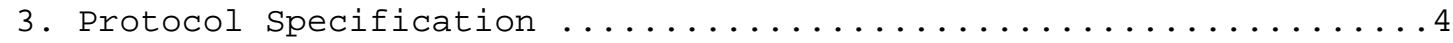

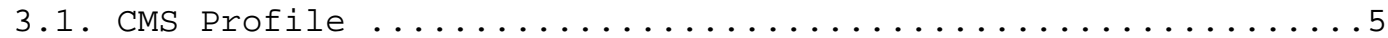

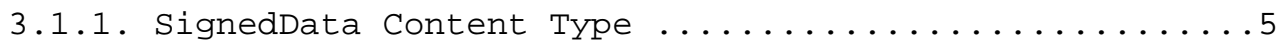

3.1.2. CMS Object Validation ......................

3.1.3. ASN.1 Specification of the CMS Signed Object ......12

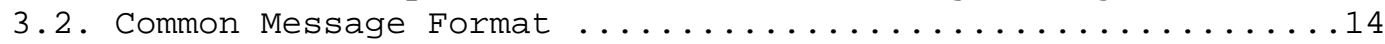

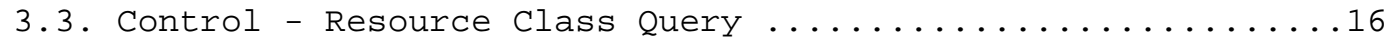

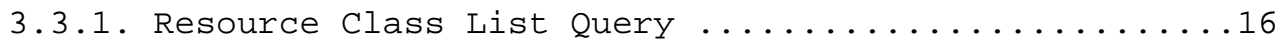

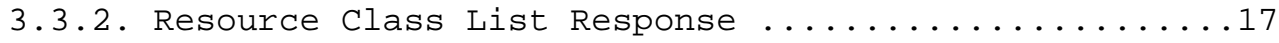

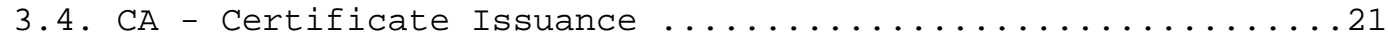

3.4.1. Certificate Issuance Request ................21

3.4.2. Certificate Issuance Response ...............24

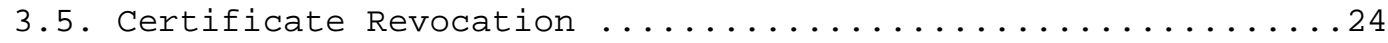

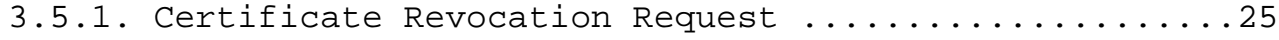

3.5.2. Certificate Revocation Response ..............26

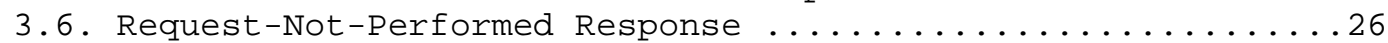

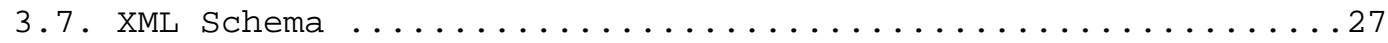

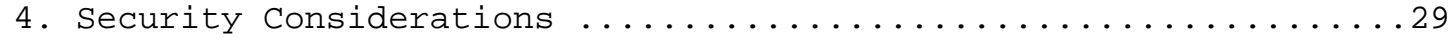

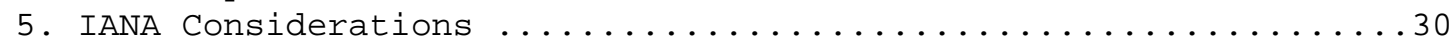

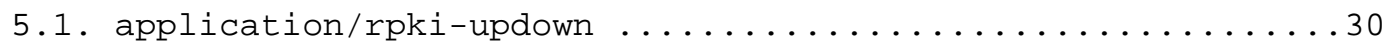

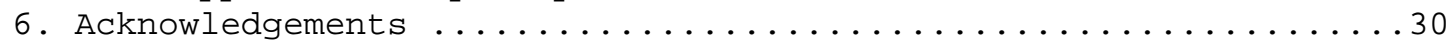

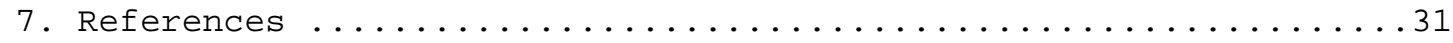

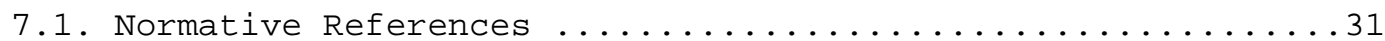

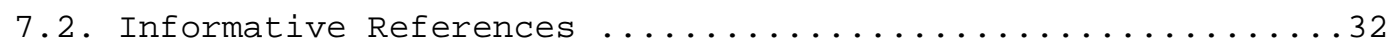




\section{Introduction}

This document defines a framework for certificate management interactions between an Internet Number Resource issuer ("issuer") and an Internet Number Resource recipient ("subject") through the specification of a protocol for interaction between the two parties. The protocol supports the transmission of requests from the subject, and corresponding responses from the issuer encompassing the actions of certificate issuance, certificate revocation, and certificate status information reports. This protocol is intended to be limited to the application of Internet Number Resource certificate management and is not intended to be used as part of a more general certificate management framework.

1.1. Terminology

Terms used in this document are:

"Internet Number Resource" (or "resource") used in the context of this document to refer to Autonomous system (AS) numbers, IP version 4 addresses, and IP version 6 addresses.

"issuer" used in the context of this document as an entity undertaking the role of resource issuer. An "issuer" is a Certification Authority (CA), and can issue resource certificates.

"subject" used in the context of this document as an entity undertaking the role of resource recipient who is the subject of a resource certificate. A "subject" may be issued with a CA-enabled certificate, allowing the entity to also assume the role of an "issuer".

"resource class" a resource class refers to a collection of resources that can be certified in a single resource certificate by an issuer.

"server" in the context of this client/server protocol specification, the issuer assumes the role of the "server".

"client" in the context of this client/server protocol specification, the subject assumes the role of the "client".

The key words "MUST", "MUST NOT", "REQUIRED", "SHALL", "SHALL NOT", "SHOULD", "SHOULD NOT", "RECOMMENDED", "MAY", and "OPTIONAL" in this document are to be interpreted as described in [RFC2119]. 


\section{Scope}

This Resource Public Key Infrastructure (RPKI) certificate provisioning protocol defines a basic set of interactions that allow a subject to request certificate issuance, revocation, and status information from the issuer, and for an issuer to maintain an issued certificate set that is aligned to the allocation records relating to each subject. The issuer's resource allocation database is the authoritative source of what resource allocations the issuer may certify for a subject.

A resource recipient (subject) may also undertake the role of a resource issuer (issuer).

This protocol specification does not encompass:

- signing of objects with keys that are certified by resource certificates, nor the issuance of end-entity certificates.

- the specification of interaction with the issuer's resource allocation database, nor the specification of a protocol to manage the publication repository.

o the interactions between client and server that establish identities, or the exchange of the certificates and validation Public Key Infrastructure (PKI) contexts used in the Cryptographic Message Syntax (CMS) [RFC5652] message exchange.

o the interactions between client and server that allow respective local CMS signing time values to be reset to mutually recognized values.

3. Protocol Specification

This RPKI certificate provisioning protocol is expressed as a simple request/response interaction, where the client passes a request to the server, and the server generates a corresponding response.

The protocol is implemented as an exchange of messages.

Messages are passed over an HTTP [RFC2616] end-to-end connection. A message exchange commences with the client initiating an HTTP POST with content type of "application/rpki-updown" and the message object as the body. The server's response is similarly an HTTP response, with the message object carried as the body of the response and with a response content type of "application/rpki-updown". The content of the POST and the server's response are "well-formed" CMS [RFC5652] objects, encoded using the Distinguished Encoding Rules (DER) for 
ASN.1 [X.509-88], formatted in accordance with the CMS profile specified in the following section. CMS is used as the signing format to sign the message object. The CMS message includes an endentity (EE) certificate that is to be used to validate the CMS digital signature (see Section 3.1.1.4); the certificate chain that is used to validate the EE certificate MAY be included in the CMS message, and if it is not present it is assumed to have been communicated between the two entities, through mechanisms not defined in this specification.

The protocol's request/response interaction is assumed to be reliable, in that all requests MUST generate a matching response. The protocol requires sequential operation for each distinct client, where the server MUST NOT accept a client's request unless it has generated and sent a response to the client's previous request. Attempts by the client to initiate multiple requests in parallel (i.e., multiple concurrent requests with a common sender attribute (see Section 3.2) in the request) MUST be detected by the server and rejected with an error response (i.e., an error code 1101 response).

\subsection{CMS Profile}

The format of the CMS object is:

ContentInfo : := SEQUENCE \{

content Type ContentType,

content [0] EXPLICIT ANY DEFINED BY contentType \}

ContentType : := OBJECT IDENTIFIER

The content-type is the signed-data type of id-data, namely id-signedData, OID = 1.2.840.113549.1.7.2. [RFC5652]

\subsubsection{SignedData Content Type}

According to the CMS standard [RFC5652], signed-data content types are the ASN.1 type SignedData:

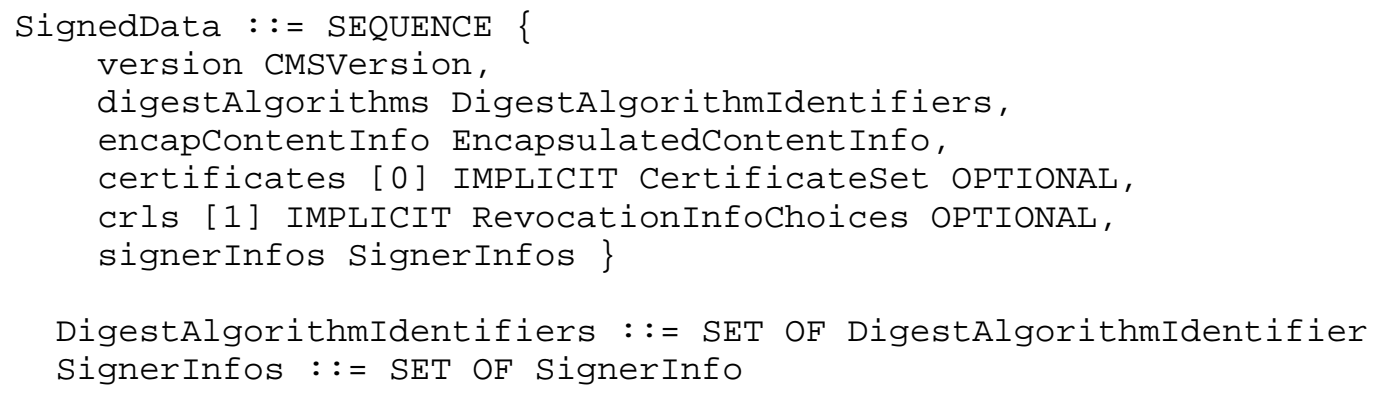


Additionally, the SignerInfos set MUST contain only a single SignerInfo object.

3.1.1.1. version

The version is the syntax version number. It MUST be 3, corresponding to the signerInfo structure having version number 3 .

\subsubsection{2. digestAlgorithms}

The digestAlgorithms set contains the Object Identifiers (OID)s of the digest algorithm(s) used in signing the encapsulated content. This set MUST contain exactly one digest algorithm OID, and the OID MUST be selected from those specified in [RFC6485].

\subsubsection{3. encapcontentInfo}

encapcontentInfo is the signed content, consisting of a content type identifier and the content itself. The encapcontentInfo represents the payload of the RPKI certificate provisioning protocol.

EncapsulatedContentInfo : := SEQUENCE \{ econtent Type Content Type, econtent [0] EXPLICIT OCTET STRING OPTIONAL \}

ContentType : := OBJECT IDENTIFIER

3.1.1.3.1. econtent Type

The eContentType for the RPKI Protocol Message object is defined as id-ct-xml, and has the numerical value of 1.2.840.113549.1.9.16.1.28. id-smime OBJECT IDENTIFIER : := \{ iso(1) member-body(2) us (840) rsadsi(113549) pkcs(1) pkcs9(9) 16 \}

id-ct OBJECT IDENTIFIER $::=\{$ id-smime 1$\}$

id-ct-xml OBJECT IDENTIFIER : := \{id-ct 28$\}$

3.1.1.3.2. eContent

The content of an RPKI XML Protocol Object consists of a single protocol message, structured according to a defined XML schema, as defined in subsequent sections of this document. The econtent field of the CMS object is formally defined using ASN.1 as:

RPKIXMLProtocolobject : := OCTET STRING -- XML encoded message 


\subsubsection{4. certificates}

This field MUST be present and MUST contain the single EE certificate of the key pair whose private key value was used to sign the CMS. This MUST NOT be an RPKI certificate, and SHOULD be a certificate that is recognized to attest to the identity of the party that created the CMS object.

This field MAY contain CA certificates that a relying party MAY use to validate the EE certificate.

3.1.1.5. crls

This field MUST be present. The contents of the field are specified in [RFC5652]. The current Certificate Revocation List (CRL) issued by the same CA that issued the EE certificate of the key pair whose private key value was used to sign the CMS MUST be present in this field. This field MAY contain CRLs issued by other CAs covering CA certificates included in the certificates field of the CMS object (see section 3.1.1.4). This field MUST NOT contain any other CRLs.

\subsubsection{SignerInfo}

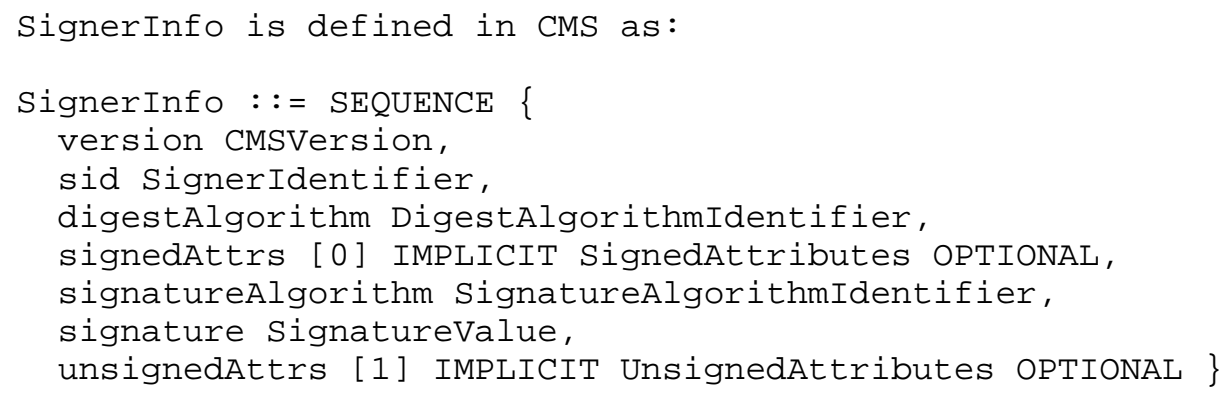

3.1.1.6.1. version

The version number MUST be 3, corresponding with the choice of SubjectKeyIdentifier for the sid.

$3.1 .1 .6 .2 . \operatorname{sid}$

The sid is defined as:

SignerIdentifier : := CHOICE \{ issuerAndSerialNumber IssuerAndSerialNumber, subjectKeyIdentifier [0] SubjectKeyIdentifier \} 
In this profile, the sid MUST be the SubjectKeyIdentifier that appears in the EE certificate carried in the CMS certificates field.

3.1.1.6.3. digestAlgorithm

The digestAlgorithm MUST consist of the OID of a digest algorithm that conforms to the RPKI Algorithms and Key Size Profile specification [RFC6485].

\subsubsection{4. signedAttrs}

The signedAttrs field is defined as:

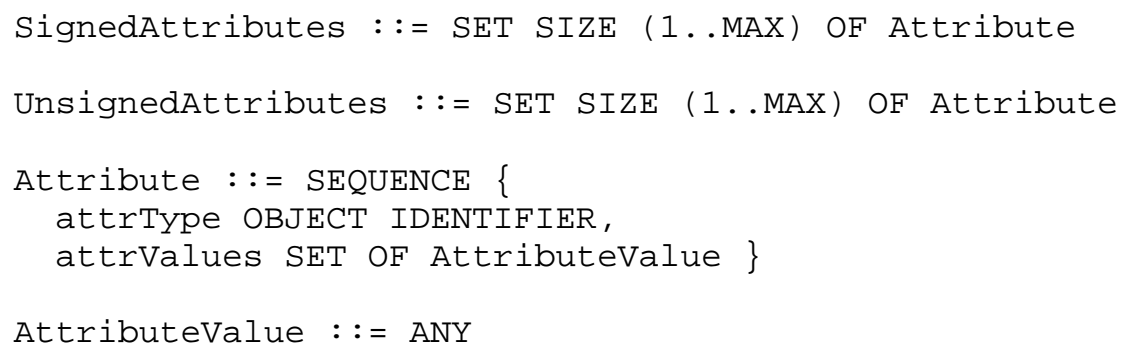

The signedAttr element MUST be present and MUST include the contenttype and message-digest attributes [RFC5652]. If either the signingtime [RFC5652] attribute or the binary-signing-time attribute [RFC6019], or both attributes, are present, they MUST also be included as the SignedAttributes. Other signed attributes MUST NOT be included.

The signedAttr MUST include only a single instance of any particular attribute. Additionally, even though the syntax allows for a SET OF Attributevalue, in this profile, the attrValues MUST consist of only a single AttributeValue.

3.1.1.6.4.1. Content-Type Attribute

The content-type attribute MUST be present. The attrType OID for the content-type attribute is 1.2.840.113549.1.9.3.

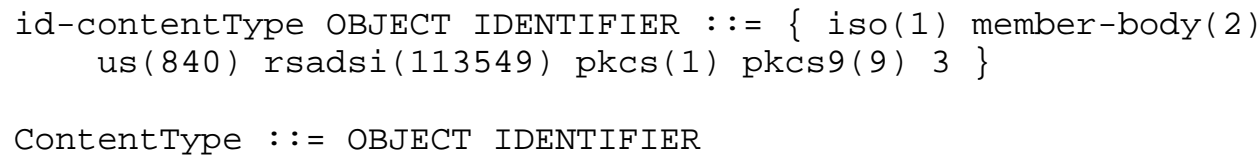


The attrValues for the content-type attribute MUST match the eContentType in the EncapsulatedContentInfo. This OID value is defined as id-ct-xml and has the numerical value of 1.2 .840 .113549 .1 .9 .16 .1 .28 .

\subsection{Message-Digest Attribute}

The message-digest attribute MUST be present. The attrType OID for the message-digest attribute is 1.2.840.113549.1.9.4.

id-messageDigest OBJECT IDENTIFIER ::= \{ iso(1) member-body (2) us (840) rsadsi(113549) pkcs(1) $\operatorname{pkcs9}(9) \quad 4\}$

MessageDigest : := OCTET STRING

The attrValues for the message-digest attribute contains the output of the digest algorithm applied to the content being signed, as specified in Section 5.4 of [RFC5652].

\subsection{Signing-Time Attribute}

The signing-time attribute MAY be present. The attrType OID for the signing-time attribute is 1.2.840.113549.1.9.5.

id-signingTime OBJECT IDENTIFIER : := \{ iso(1) member-body (2) us (840) rsadsi(113549) pkcs(1) pkcs9(9) 5$\}$

SigningTime : := Time

Time : := CHOICE \{

utcTime UTCTime, generalizedTime GeneralizedTime \}

The signing-time attribute specifies the time, based on the local system clock, when the digital signature was applied to the content. Guidelines regarding the use of UTCTime and GeneralizedTime in the signing-time attribute can be found in section 11.3 of [RFC5652].

Either one of the signing-time attribute or the binary-signing-time attribute, or both attributes, MUST be present. If both the signingtime and binary-signing-time attributes are present, they MUST both represent the same underlying time value. 


\subsection{Binary-Signing-Time Attribute}

The binary-signing-time attribute MAY be present. The attrType OID

for the binary-signing-time attribute is 1.2.840.113549.1.9.16.2.46.

$$
\begin{aligned}
& \text { id-aa-binarySigningTime OBJECT IDENTIFIER }::=\{\text { iso(1) } \\
& \text { smime (16) aa(2) } 46\} \\
& \text { BinarySigningTime : := BinaryTime } \\
& \text { BinaryTime : := INTEGER }(0 \ldots \text { MAX) }
\end{aligned}
$$$$
\text { member-body (2) us (840) rsadsi(113549) pkcs(1) pkcs9(9) }
$$

The binary-signing-time attribute specifies the time, based on the local system clock, when the digital signature was applied to the content. The precise definition of the binary-signing-time attribute can be found at [RFC6019].

Either one of the signing-time or the binary-signing-time attributes, or both attributes, MUST be present. If both the signing-time and binary-signing-time attributes are present, they MUST both represent the same underlying time value.

3.1.1.6.5. signatureAlgorithm Attribute

The signatureAlgorithm MUST conform to the RPKI Algorithms and Key Size Profile specification [RFC6485].

3.1.1.6.6. signature Attribute

The signature value is defined as:

SignatureValue : := OCTET STRING

The signature characteristics are defined by the digest and signature algorithms.

\subsection{UnsignedAttributes Attribute}

unsignedAttrs MUST be omitted.

\subsubsection{CMS Object Validation}

Before a recipient of a CMS signed object can use the content of the object, the recipient MUST validate the signed object by verifying that all of the following conditions hold. A recipient may perform these checks in any order. 
1. The CMS object is well formed, such that the signed object syntax complies with this specification. In particular, that each of the following is true:

a. The content-type of the CMS object is SignedData (OID $1.2 .840 .113549 .1 .7 .2)$

b. The version of the signedData object is 3 .

c. The certificates field in the signedData object is present and contains one EE certificate, the SubjectKeyIdentifier field of which matches the sid field of the SignerInfo object.

d. The crls field in the SignedData object is present.

e. The version of the SignerInfo is 3 .

f. The signedAttrs field in the SignerInfo object is present and contains one each of the content-type attribute (OID 1.2.840.113549.1.9.3), the message-digest attribute (OID 1.2.840.113549.1.9.4), and either or both of a single instance of the signing-time attribute (OID 1.2.840.113549.1.9.5) and the binary-signing-time attribute (OID 1.2.840.113549.1.9.16.2.46), and no other attributes.

g. The eContentType in the EncapsulatedContentInfo is an OID that matches the attrvalue in the content-type attribute and has the attrValue of id-ct-xml.

h. The unsignedAttrs field in the SignerInfo object is omitted.

i. If both the signing-time attribute and the binary-signingtime attribute are present, then their values represent the same time.

j. The digestAlgorithm in the SignedData and SignerInfo objects conforms to the RPKI Algorithms and Key Size Profile specification [RFC6485].

k. The signatureAlgorithm in the SignerInfo object conforms to the RPKI Algorithms and Key Size Profile specification [RFC6485].

1. The signed object is DER encoded. 
2. The public key of the EE certificate (contained within the CMS signed-data object) can be used to successfully verify the signature on the signed object.

3. The EE certificate (contained within the CMS signed-data object) is a valid EE certificate. In particular, there exists a valid certification path from a trust anchor selected by the recipient to this EE certificate.

4. At the current time, the EE certificate is not revoked. This can be determined by confirming that the CRL contained in the crls field of the CMS signed data object is a current valid CRL, issued by the same CA that issued the EE certificate, and the CRL does not list the serial number of the EE certificate.

5. The time represented by the signing-time attribute or the binarysigning-time attribute is greater than or equal to the time value passed in previously valid CMS objects that were passed from the same originator to this recipient. This signing time value MAY lie within the Validity Time of the EE certificate, but the EE certificate SHOULD NOT be considered invalid if this is not the case when all other checks listed here are passed.

3.1.3. ASN.1 Specification of the CMS Signed Object

The following is the ASN.1 specification of the CMS signed object used by the RPKI provisioning protocol.

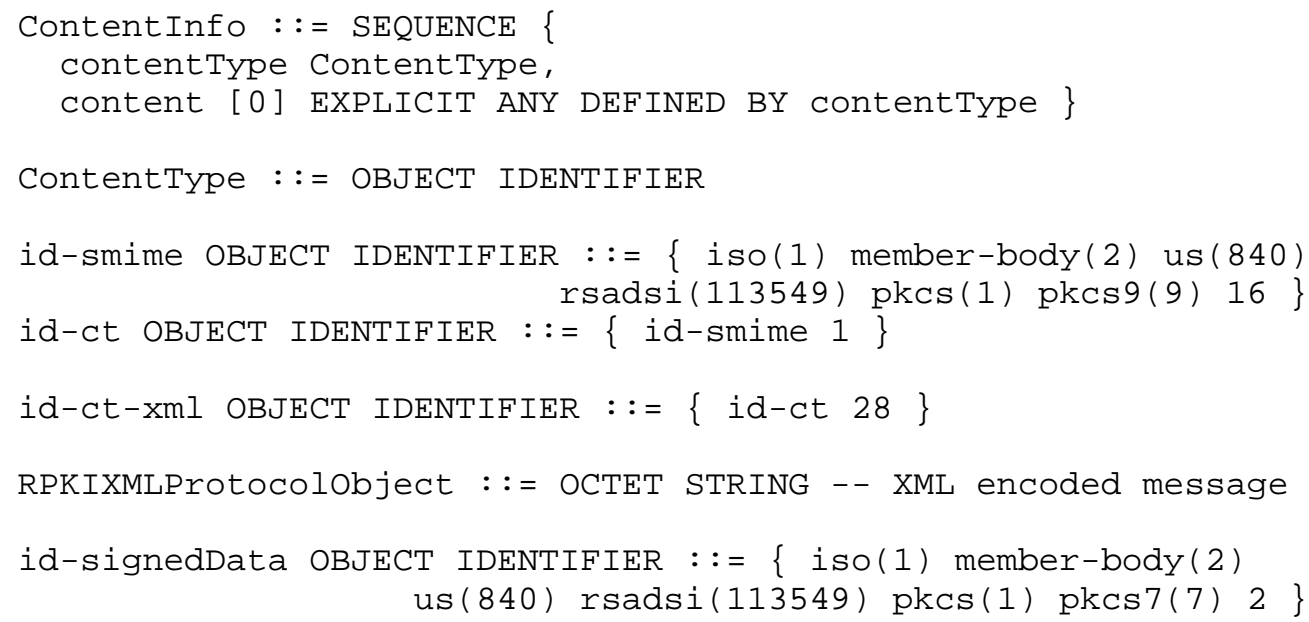




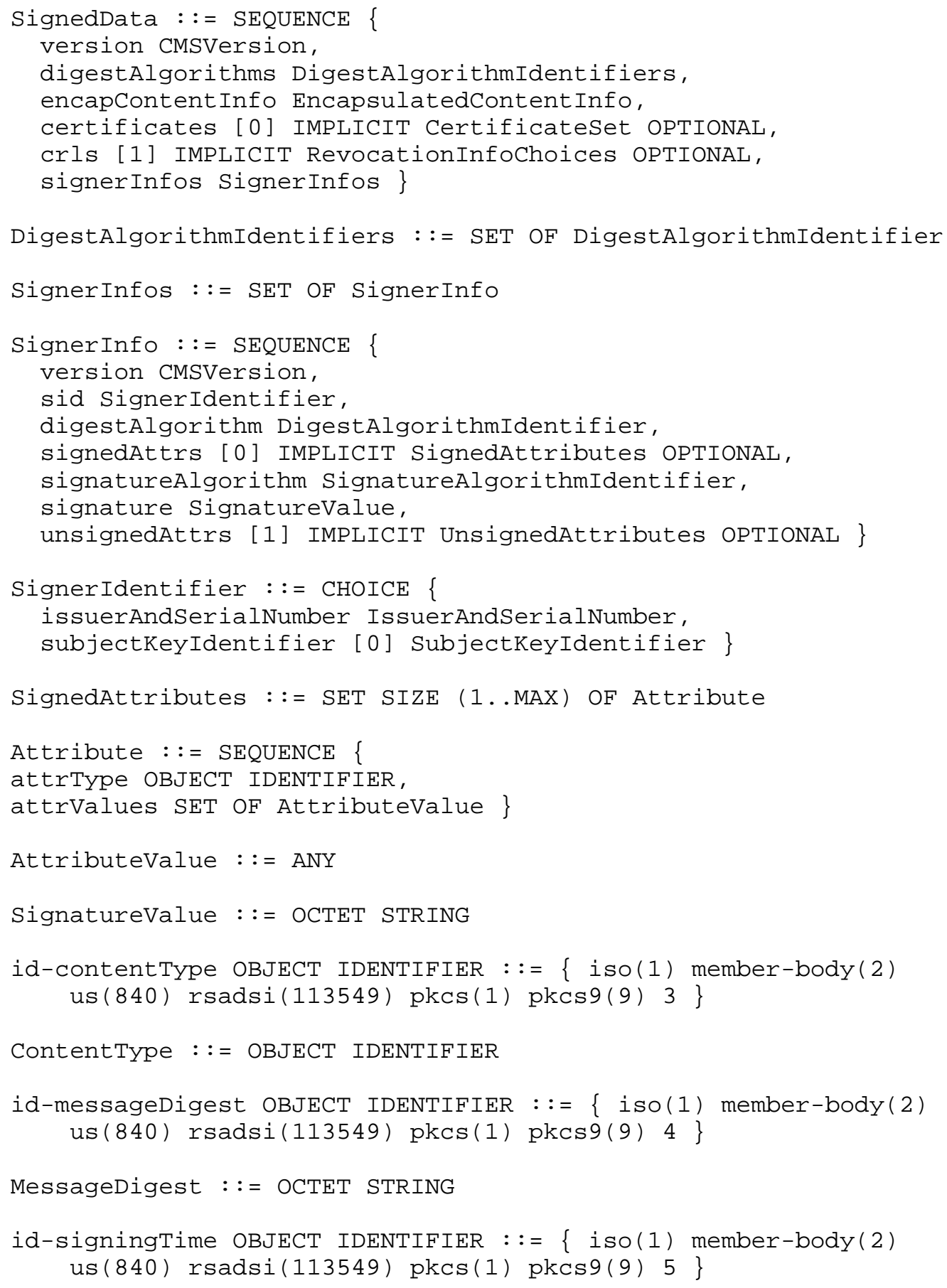




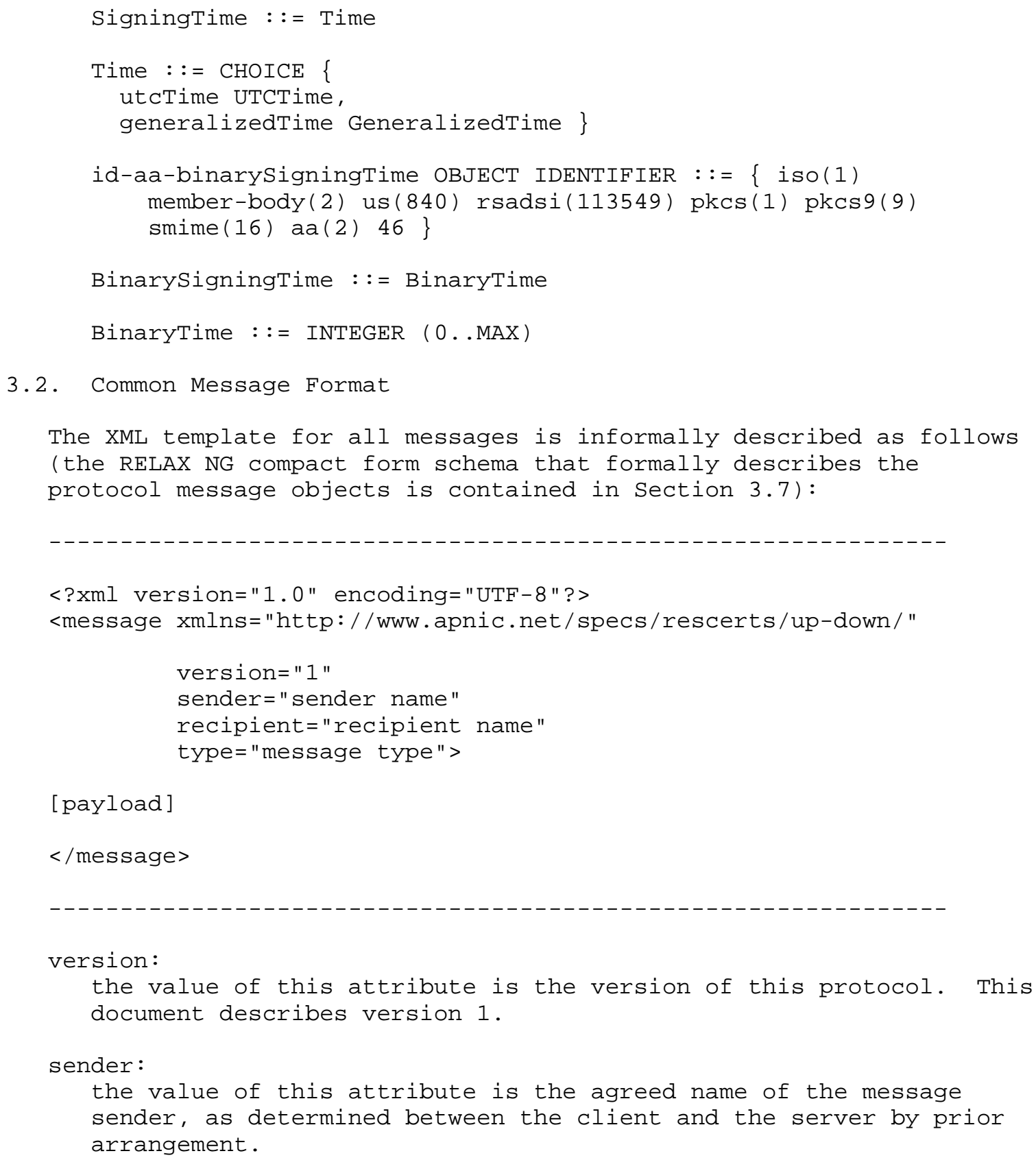


recipient :

the value of this attribute is the agreed name of the message recipient, as determined between the client and the server by prior arrangement.

type:

the possible values of this attribute are "list", "list_response", "issue", "issue_response", "revoke", "revoke_response", and

"error_response".

Conforming parsers MUST reject any document with a version number they do not understand or with any elements or attributes they do not understand. Servers must generate an error response when receiving such a request. Clients should generate an error when receiving such a response.

The encapsulated content of the CMS wrapping is an XML document. The remainder of this protocol specification omits this CMS wrapper and only discusses the XML document.

Messages are checked using the following tests:

1. Check that the CMS is well-formed (see test 1 of section 3.1.2).

2. Check that the XML is well-formed.

3. Check that the XML sender and recipient attributes reference a known client and this server's system respectively for a query, and the previously addressed server and this client for a response.

4. Verify the digital signature using the public key provided in the certificate carried in the CMS wrapper (see test 2 of section 3.1.2).

5. Validate the CMS-provided certificate using the PKI that has been determined by prior arrangement between the client and server (see test 3 of Section 3.1.2).

6. Check that the signing time of the CMS is equal to or greater than the signing time provided in the most recent previous message that this recipient has received from this sender (see test 4 of Section 3.1.2).

7. Check that the value of the version number of the message is 1 .

These checks SHOULD be applied in the order specified here. 
Any errors encountered while checking items 1 through 7 MUST cause a server to generate an "HTTP 400 Bad Request" response to the HTTP POST operation. An error in step 7 MUST cause the server to generate a "Request-Not-Performed" error response. Any errors encountered in these tests by a client SHOULD cause the client to generate an error.

A server MAY perform flow control on the rate of processed requests. Requests not processed due to such a flow control constraint MAY cause the server to generate an "HTTP 503 Service Unavailable" response. An HTTP 503 response MAY include an HTTP Retry-After: header as a hint to the client.

\subsection{Control - Resource Class Query}

This query is used for a client to query a server for a list of all resources that have been allocated or assigned by the server to the client. In addition, the server's response will contain a copy of the current certificates issued by the server's CA where this client is the certificate's subject.

\subsubsection{Resource Class List Query}

The value of the message "type" message attribute for this query is:

type="list"

Payload:

[No message payload is defined for this query] 


\subsubsection{Resource Class List Response}

The value of the message "type" element for this response is:

type="list_response"

Payload:

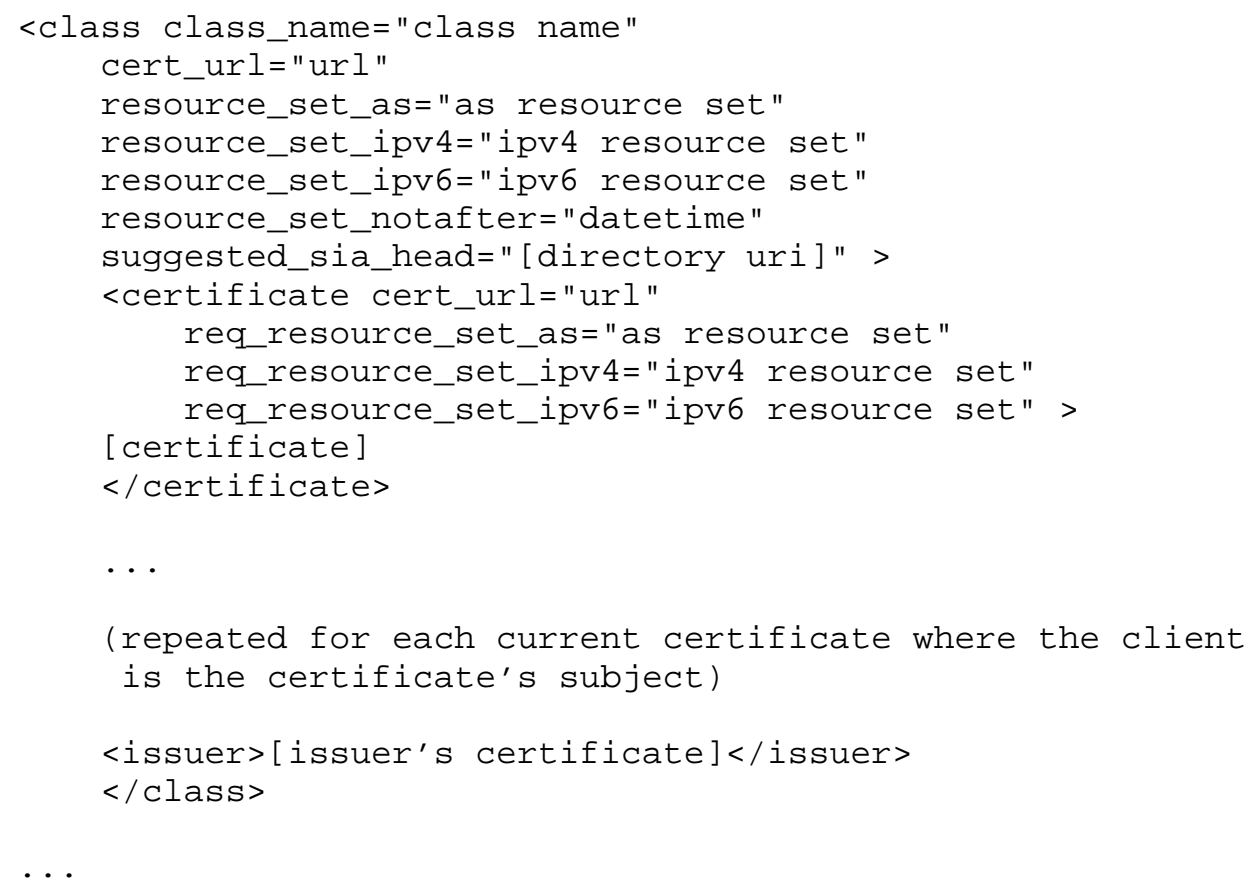

(repeated for each of the issuer's resource class where the client has been allocated resources)

Where the client has been allocated resources from multiple resource classes, the response MUST contain multiple class elements that correspond to the complete set of the issuer's resource classes where the client holds allocated resources. Those issuer's resource classes where the client holds no allocated resources MUST NOT be included in the response.

Where the issuer has issued multiple certificates in a resource class signed with different keys (as may occur during a staged issuer-key 
rollover), only the most recent certificate issued with the currently "active" issuer's key is to be listed in the response.

Each "class" element describes a set of resources that are certified within the scope of a single certificate, referring to a single resource class with a common validation path.

class_name:

the value of this attribute is the issuer-assigned name of the issuer's resource class.

cert_url:

in the context of a class element, the value of this attribute is a pointer to the issuer's CA certificate (i.e., a reference to the immediate superior certificate, being the CA-enabled certificate where the issuer is the certificate's subject). Its value is a comma-separated list of URIs, of which at least one MUST be an rsync URI [RFC5781]. Any comma values within a URI MUST be escaped ("이). The ordering of the list may be interpreted by the client as a relative preference for access methods as expressed by the publisher of this certificate.

resource_set_as:

in the context of a class element, the value of this attribute is the set of AS numbers and AS number ranges that the issuer has allocated to the client within the scope of this resource class, presented in ASCII as a comma-separated list. The list elements are decimal integer values and ranges of decimal integers specified by the lowest and highest values of the range with a hyphen delimiter, using the canonical order as described in [RFC3779], without leading zeros, and with no white space or punctuation other than the comma and the hyphen range designator (e.g., resource_set_as="123,456-789,123456"). If there are no AS numbers in this resource class, then the empty AS set is represented by a null string value (") for this attribute.

resource_set_ipv4:

in the context of a class element, the value of this attribute is the set of IPv4 addresses that the issuer has allocated to the client within the scope of this resource class. The value is presented in ASCII as a comma-separated list of elements. Each element is either an address prefix using the notation of <dotted quad $>/$ mask length, or a range specified as the lowest and highest values of the range in dotted quad notation with a hyphen delimiter. The list is presented in canonical order, as described in [RFC3779]. The dotted quad notation is without leading zeros, and the list contains no white space or punctuation other than the period, forward slash, hyphen, and comma (e.g., 
resource_set_ipv4="192.0.2.0/26,192.0.2.66-192.0.2.76"). If there are no IPv4 addresses in this resource class, the empty IPv4 address set is represented by a null string value ("") for this attribute.

resource_set_ipv6:

in the context of a class element, the value of this attribute is the set of IPv6 addresses that the issuer has allocated to the client within the scope of this resource class. The value is presented in ASCII as a comma-separated list of elements. Each element is either an address prefix using the notation of <hex nibble sequence>/mask length, or a range specified as lowest and highest values of the range in hex nibble notation with a hyphen delimiter. Trailing zero nibbles are truncated and represented by '::'. The list is presented in canonical order, as described in [RFC3779]. The hex nibble sequence notation is without leading zeros, and the list contains no white space or punctuation other than the colon, forward slash, hyphen, and comma, and conforms to the canonical format of [RFC5952] (e.g., resource_set_ipv6="2001:db8::/48,2001:db8:2::-2001:db8:5::"). The XML Schema data type is "http://www.w3.org/TR/xmlschema-2/\#hexBinary" and the value is case insensitive, with the canonical form being lower case. If there are no IPv6 addresses in this resource class, the empty IPv6 address set is represented by a null string value (") for this attribute.

resource_set_notafter:

The value of this attribute specifies the date/time that would be set in the Validity notAfter field in any new certificate issued for this particular client within the scope of this resource class, should the client request a new certificate. The time format used for the value of this attribute is specified as defined in ISO 8601 [ISO.8601:2004], and MUST use UTC time represented as YYYY-MM-DDThh:mm:ssZ (e.g., 2007-11-29T04:40:00Z). If the client's certificate has a validity notAfter time that is different from this time, then the client SHOULD request a new certificate to be issued for this resource class.

suggested_sia_head: (OPTIONAL) If this field is present, then its value is a directory URI that indicates a repository publication point that the server has made available to the client to use for the client's collection of published products. This specification does not encompass the protocols that the client may use with the operator of the repository publication point in order to publish objects at this publication point. 
[issuer's certificate]

value is the Base64 encoding of the DER-encoded issuer's CA certificate (the CA-enabled certificate where the issuer is the certificate's subject).

Each certificate element describes the most recently issued current certificate where the certificate's subject refers to the client for each active client key pair. A "current" certificate is a non-expired, non-revoked certificate. If no current certificate has been issued, then no certificate element is to be included in the response.

cert_url:

in the context of a certificate element, this is a pointer to the location where the certificate issuer has published this certificate. This field is the issuer's suggestion for the Authority Information Access (AIA) field for the subject to use in subordinate certificates that are issued by the subject. According to the Resource Certificate Profile [RFC6487], the AIA field is a non-empty (contains a minimum of 1 element) list of URI's, one of which MUST be an rsync URI [RFC5781]. The order of URI's in the AIA field may be interpreted as the publisher's relative preference for access methods for this certificate. The cert_url conforms to this AIA specification. Its value is a comma-separated list of URIs, one of which MUST be an rsync URI. Any comma values within a URI MUST be escaped ("2C").

req_resource_set_as:

the set of AS numbers that were specified in the corresponding original certificate request that defined the maximal requested span of the certified AS number set, following the syntax described above. If this attribute was present in the certificate request, then the attribute MUST be present in this response; otherwise, it MUST NOT be present.

req_resource_set_ipv4:

the set of IPV4 addresses that were specified in the corresponding original certificate request that defined the maximal requested span of the certified IPv4 address set, following the syntax described above. If this attribute was present in the certificate request, then the attribute MUST be present in this response; otherwise, it MUST NOT be present.

req_resource_set_ipv6:

the set of IPV6 addresses that were specified in the corresponding original certificate request that defined the maximal requested span of the certified IPv6 address set, following the syntax described above. If this attribute was present in the certificate 
request, then the attribute MUST be present in this response; otherwise, it MUST NOT be present.

[certificate]

value is the Base64 encoding of the DER-encoded certificate.

3.4. CA - Certificate Issuance

This query is used by the client to request the server's CA to issue a resource certificate for the resources that have been allocated or assigned to the client. If the request can be successfully processed, then the server's response includes the issued certificate.

3.4.1. Certificate Issuance Request

The value of the message "type" element for this request is:

type="issue"

Payload:

<request

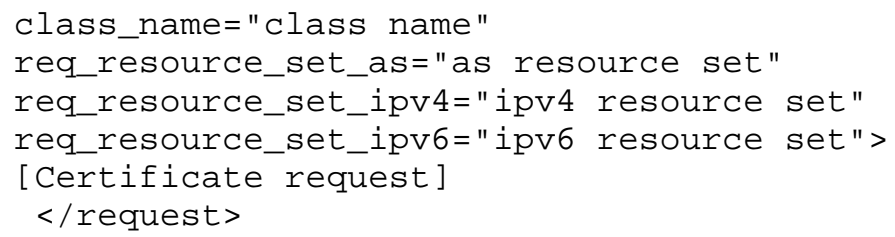

The client MUST use different key pairs for each distinct resource class.

The req_resource_set attributes are optional in the request.

If none of the req_resource_set attributes are specified, then the request signifies that the complete set of all resources that match the client's current resource allocation is to be included in the issued certificate.

If any of the req_resource_set attributes are specified in the request, then any missing req_resource_set attributes are to be interpreted as specifying the complete set of the corresponding 
resource type that match the client's current resource allocation are to be included in the issued certificate.

If the value of any included req_resource_set attributes is the null value (""), then this indicates that no resources of that resource type are to be included in the issued certificate.

The requested resource set values are held as a local record by the issuer against the resource class and the client's public key. Any subsequent Certificate Issuance Requests that specify the same resource class and the same client's public key will (re) set the issuer's local record of the requested resource sets to the most recently specified values.

class_name:

value is the server's identifier of a resource class.

req_resource_set_as: (OPTIONAL)

the set of AS numbers that define the maximal requested span of the certified AS number set, formatted as per the resource_set_as attribute of the resource class list response.

req_resource_set_ipv4: (OPTIONAL) the set of IPv4 addresses that define the maximal requested span of the certified IPv4 address set, formatted as per the resource_set_ipv4 attribute of the resource class list response.

req_resource_set_ipv6: (OPTIONAL) the set of IPV6 addresses that define the maximal requested span of the certified IPv6 address set, formatted as per the resource_set_ipv6 attribute of the resource class list response.

[Certificate request] value is the certificate request. This is a Base64 encoded DER version of a request formatted using PKCS\#10 [RFC2986]. The certificate request is signed using the private key part of the key pair whose public part is the subject key value in the certification request. The signing algorithm is specified in [RFC6485]. (This signature component is intended to demonstrate proof of possession of the private key.)

The response to this request is a Certificate Issuance Response if the request can be processed online. If the request cannot be undertaken immediately, then the server MUST respond with a "RequestNot-Performed" message, using the appropriate error code:

- If the resource class is not defined by the server, then the server MUST return error code 1201 . 
- If the client holds no resources in a defined resource class, then the server MUST return error code 1202 and not proceed with the request.

- If the certificate request payload is badly formed, then the server MUST return error code 1203 .

- If the public key used in the certificate request implies that the client is attempting to use identical key pairs for multiple resource classes, then the server MUST respond with a 1204 error code.

- If the certificate issuer uses an off-line process to undertake certificate issuance, and the server cannot directly respond to the certificate issuance request with an issued certificate, then the certificate issuer MUST respond to the first instance of this request with an error code 1104 to indicate that the request is being processed asynchronously. Subsequent repetitions of this request while the off-line actions are being undertaken SHOULD cause a response with error code 1101. In this context, where off-line processes are invoked for certificate issuance, if the certificate issuer determines in processing the request that the issued certificate would be identical in all respects to the most recently issued certificate for this client, other than the certificate's serial number, were the certificate to be issued, the issuer may choose to respond with the most recently issued certificate and not initiate an off-line certificate issuance request.

Note that a client, when receiving a 1104 response to a certificate issuance request, MAY periodically resubmit the request, in which case the client MUST receive an error code 1101 response while the request is being processed, and a Certificate Issuance Response when the certificate issuance process has completed. In such circumstances, a client SHOULD limit the frequency of such repeated requests to no more than 1 request in each 24 -hour interval. 


\subsubsection{Certificate Issuance Response}

The value of the message "type" element for this response is:

type="issue_response"

Payload:

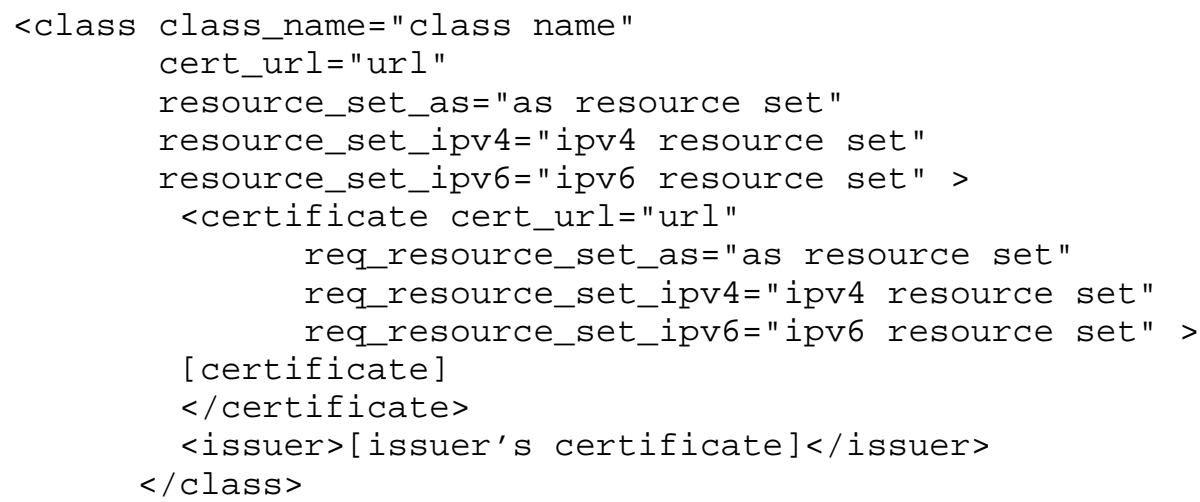

If the certificate issuer determines that the issued certificate would be identical in all respects to the most recently issued certificate for this client, other than the certificate's serial number, were the certificate to be issued, the issuer may choose to respond with the most recently issued certificate and not issue a new certificate for this request.

The definition of the attributes and syntax of the values is the same as the resource class list response, but the response only references the (single) named resource class, and the (single) certificate issued against the client's public key as provided in the corresponding certificate request.

\subsection{Certificate Revocation}

This request 'retires' a client's key pair by requesting that the server's CA revoke all certificates for this client (i.e., where this client is the subject) that contain the matching public key, within the scope of a named resource class. Individual certificates cannot be revoked within the scope of this protocol. 


\subsubsection{Certificate Revocation Request}

The value of the message "type" element for this request is:

type="revoke"

Payload :

<key class_name="class name" ski="[encoded hash of the subject public key]" />

This command directs the server's CA to immediately mark all issued valid certificates issued by this issuer within the named resource class with this client's subject name and the provided SKI value to be marked as revoked, causing the issued certificates to be withdrawn from the publication repository and to be listed in the server's subsequent CRLs within this resource class. The issuer MUST ensure that all certificates to be revoked were issued with the requesting client as the certificate's subject.

class_name:

value is the issuer-assigned name of the issuer's resource class.

ski:

value is the encoded hash of the client's public key that is to be revoked. The algorithm for the encoding is to generate the 160-bit SHA-1 hash of the client's public key, as defined in method (1) of Section 4.2.1.2 of [RFC5280], and encode this value using the Base 64 encoding with URL and Filename safe Alphabet, as defined in Section 5 of [RFC4648]. 


\subsubsection{Certificate Revocation Response}

The value of the message "type" element for this response is:

type="revoke_response"

Payload:

<key class_name="class name" ski="[encoded hash of the subject public key]" />

class_name:

value is the issuer-assigned name of the server's resource class.

ski:

value is the encoded hash of the client's public key that is to be revoked. The algorithm for the encoding is to generate the 160-bit SHA-1 hash of the client's public key, as defined in method (1) of Section 4.2.1.2 of [RFC5280], and encode this value using the Base 64 encoding with URL and Filename safe Alphabet, as defined in section 5 of [RFC4648].

3.6. Request-Not-Performed Response

The value of the message "type" element for this response is:

type="error_response"

Payload:

<status $>$ [Code] $</$ status $>$

<description xml:lang="en-US">[Readable text]</description>

All states where an error response if to be generated, either due to detected errors or inconsistencies in the content of the request or server-side states that prevent the request being performed, generate a Request-Not-Performed response. 
description:

value is a text field. This element MAY be present. It's value has no defined meaning within the scope of this protocol, and implementations may assume that some form of human-readable text may be used here. If the HTTP request that triggered this error response includes an Accept-Language header as defined in Section 14.4 of the HTTP/1.1 specification [RFC2616], then the server MAY include a second description element using the highest ranked preferred language of the client. The en-US description MUST always be included if the element is present.

The error code set is:

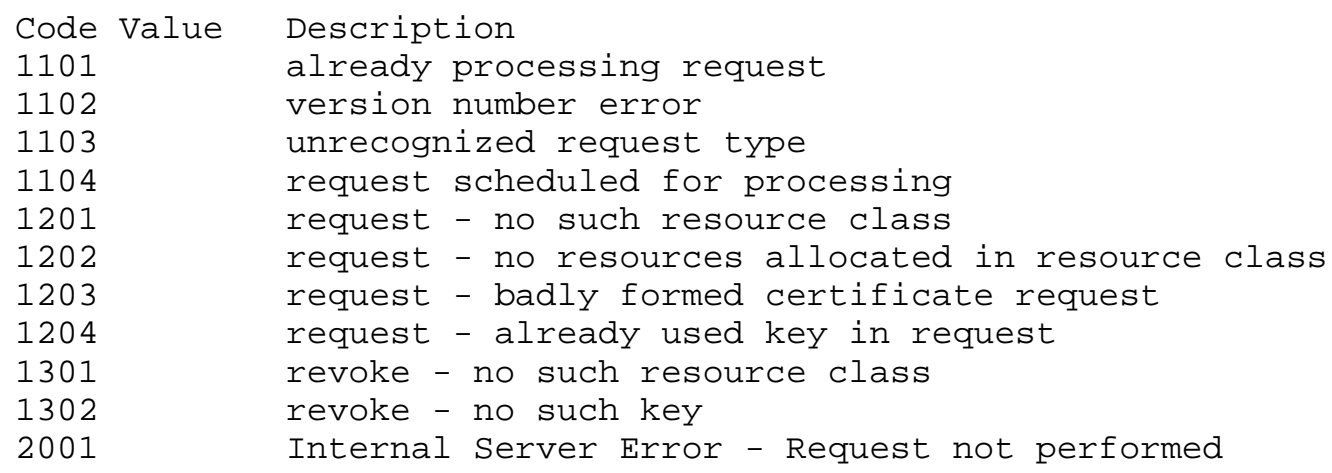

3.7. XML Schema

The following is a RELAX NG compact form schema describing version 1 of this protocol.

Note: As discussed in [XML], "the namespace name, to serve its intended purpose, SHOULD have the characteristics of uniqueness and persistence. It is not a goal that it be directly usable for retrieval of a schema (if any exists)".

default namespace = "http://www.apnic.net/specs/rescerts/up-down/"

grammar

resource_set_as $=$ xsd:string $\{$ maxLength $=" 512000 "$ pattern=" [ \-,0-9]*"

resource_set_ip4 $=$ xsd:string \{ maxLength="512000" pattern=" [\-,/.0-9]*" \}

resource_set_ip6 = xsd:string $\{$ maxLength="512000" pattern=" [\-,/:0-9a-fA-F]*"

class_name = xsd:token \{ minLength="1" maxLength="1024" \}

ski = xsd:token $\{$ minLength="27" maxLength="1024" \} 


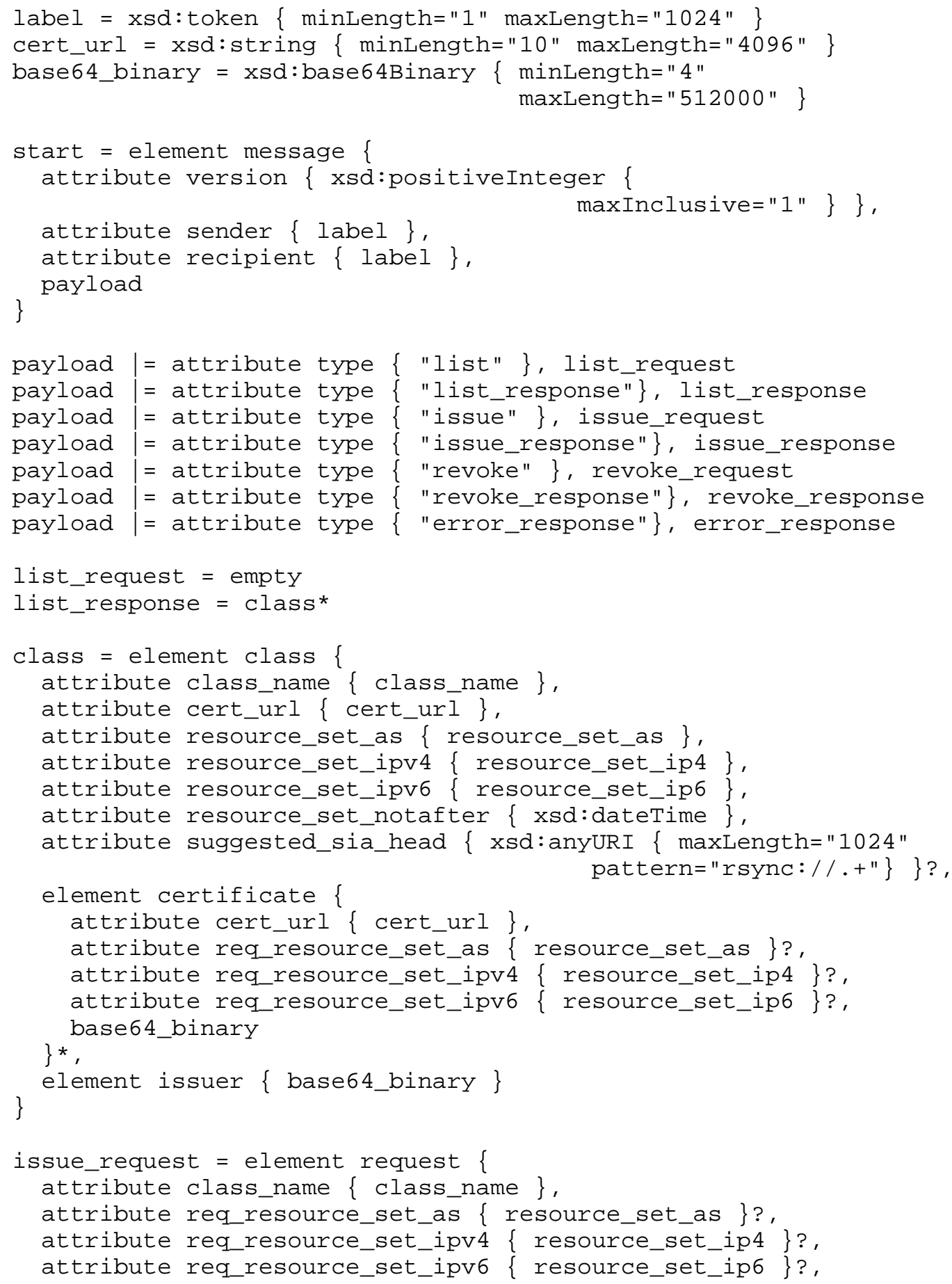




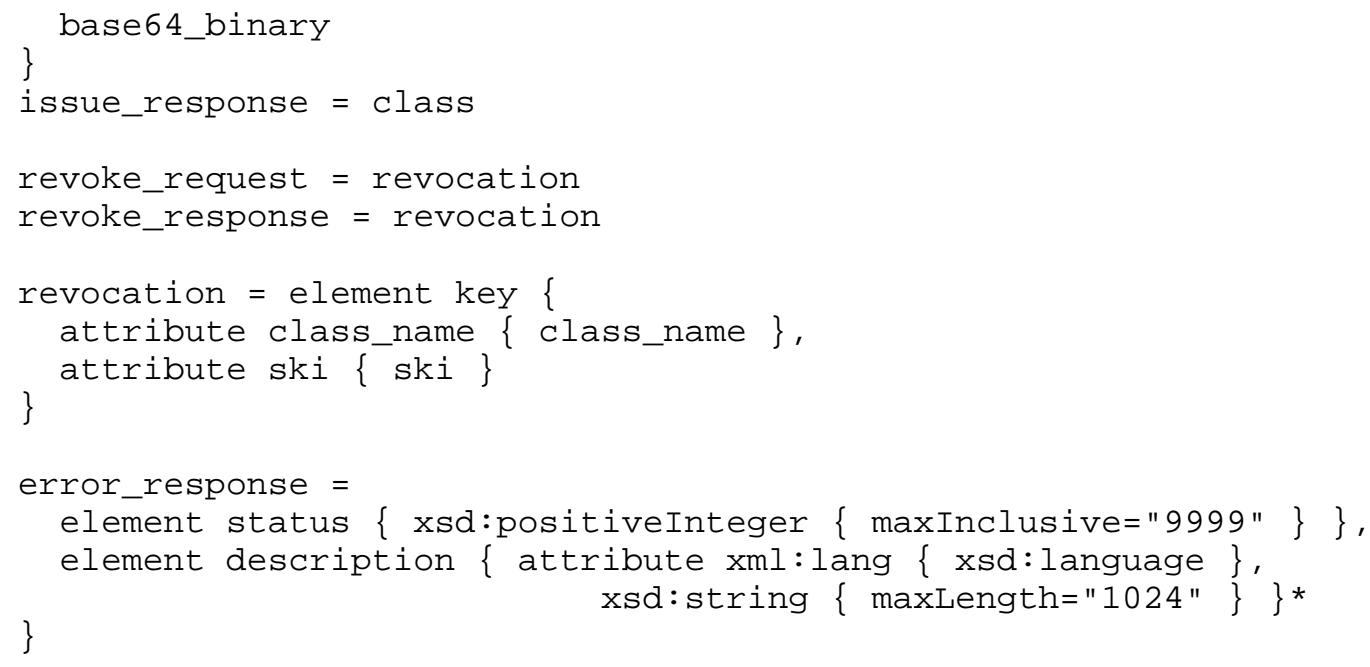

4. Security Considerations

This protocol supports the maintenance of resource certificates that the issuer issues for a subject in certifying resources that have been allocated or assigned by the issuer to the subject [RFC6480]. This protocol assumes that the issuer and subject are known to each other and have exchanged credentials so as to support the mutual recognition of the digital signatures used to sign the CMS messages. The mechanisms used to perform the associated credential exchange are not described in this specification.

The protocol is a minimal query/response protocol that imposes strict serialization on each query/response transaction, reducing the potential for the subject and the issuer to lose synchronization over the issued certificate state.

Validation of protocol objects (Section 3.1.2) requires that the CMS signing-time value be greater than or equal to the time value passed in the previously valid protocol objects that were passed from the same originator to the same recipient. If a party inadvertently sends a valid message (protocol object) with a signing time in the future, then subsequent messages from the party in the same client/server context can use signing-time value consistent with this validation constraint, such that the signing times contained in subsequent messages are greater than or equal to the signing-time value of the previous valid message. (Note that it is not a normative requirement that the signing time be precisely aligned to a time of day clock, thus permitting arbitrarily large clock skew values in the context of this protocol message exchange.) If the client and server wish to reset the signing time to a mutually agreed 
value, then, (as noted in Section 2) the interactions between the client and the server to achieve this outcome are not encompassed in this protocol.

5. IANA Considerations

IANA has registered the following media type:

application/rpki-updown

5.1. application/rpki-updown

Type name: application

Subtype name: rpki-updown

Required parameters: None

Optional parameters: None

Encoding considerations: binary

Security considerations: Carries an RPKI Provisioning Protocol Message, as defined in this document.

Interoperability considerations: None

Published specification: This document

Applications that use this media type: HTTP [RFC5652]

Additional information:

Magic number $(s)$ : None

File extension (s):

Macintosh File Type Code(s):

Person \& email address to contact for further information:

Geoff Huston <gih@apnic.net>

Intended usage: COMMON

Restrictions on usage: Only to be used as an RPKI Provisioning

Protocol message object type, as defined in this document.

Author: Geoff Huston <gih@apnic.net>

Change controller: Geoff Huston <giheapnic.net>

6. Acknowledgements

The authors would like to acknowledge the valued contributions from Russ Housley, Steve Kent, Randy Bush, George Michaelson, Robert Kisteleki, Tim Bruijnzeels, and Carsten Bormann in the preparation of the protocol described in this document. 
7. References

7.1. Normative References

[ISO.8601:2004] ISO, "ISO 8601:2004 Representation of dates and Times", 2004 .

[RFC2119] Bradner, S., "Key words for use in RFCs to Indicate Requirement Levels", BCP 14, RFC 2119, March 1997.

[RFC2616] Fielding, R., Gettys, J., Mogul, J., Frystyk, H., Masinter, L., Leach, P., and T. Berners-Lee, "Hypertext Transfer Protocol -- HTTP/1.1", RFC 2616, June 1999.

[RFC2986] Nystrom, M. and B. Kaliski, "PKCS \#10: Certification Request Syntax Specification Version 1.7", RFC 2986, November 2000 .

[RFC3779] Lynn, C., Kent, S., and K. Seo, "X.509 Extensions for IP Addresses and AS Identifiers", RFC 3779, June 2004.

[RFC4648] Josefsson, S., "The Base16, Base32, and Base64 Data Encodings", RFC 4648, October 2006.

[RFC5280] Cooper, D., Santesson, S., Farrell, S., Boeyen, S., Housley, R., and W. Polk, "Internet X.509 Public Key Infrastructure Certificate and Certificate Revocation List (CRL) Profile", RFC 5280, May 2008 .

[RFC5652] Housley, R., "Cryptographic Message Syntax (CMS) ", STD 70, RFC 5652, September 2009.

[RFC5781] Weiler, S., Ward, D., and R. Housley, "The rsync URI Scheme", RFC 5781, February 2010.

[RFC5952] Kawamura, S. and M. Kawashima, "A Recommendation for IPv6 Address Text Representation", RFC 5952, August 2010.

[RFC6019] Housley, R., "BinaryTime: An Alternate Format for Representing Date and Time in ASN.1", RFC 6019, September 2010 .

[RFC6485] Huston, G., "The Profile for Algorithms and Key Sizes for Use in the Resource Public Key Infrastructure (RPKI)", RFC 6485, February 2012 . 
[X.509-88] CCITT, "Recommendation X.509: The DirectoryAuthentication Framework", 1988.

7.2. Informative References

[RFC6480] Lepinski, M. and S. Kent, "An Infrastructure to support Secure Internet Routing", RFC 6480, February 2012.

[RFC6487] Huston, G., Michaelson, G., and R. Loomans, "A Profile for X.509 PKIX Resource Certificates", RFC 6487, February 2012 .

[XML] Bray, T., Hollander, D., Layman, A., Tobin, R., and H. Thompson, "Namespaces in XML 1.0 (Third Edition)", World Wide Web Consortium Recommendation REC-xml-names-20091208, December 2009, <http://www.w3.org/TR/2009/REC-xmlnames-20091208/>.

Authors' Addresses

Geoff Huston

APNIC

EMail: gih@apnic.net

URI: http://www.apnic.net

Robert Loomans

APNIC

EMail: robertl@apnic.net

URI : http://wWw.apnic.net

Byron Ellacott

APNIC

EMail: bjedapnic.net

URI: http://www.apnic.net

Rob Austein

Internet Systems Consortium

EMail: sra@hactrn.net 